\title{
The effects of time-out locus during fixed-ratio reinforcement
}

\author{
ELLIS I. BAROWSKY \\ Hunter College, The City University of New York, New York, New York 10031 \\ and \\ DONALD E. MINTZ \\ City College, The City University of New York, New York, New York 10031
}

\begin{abstract}
Pigeon's keypecking was reinforced on a multiple fixed-ratio schedule. All three components were fixed ratio 60; a 10-sec time-out regularly appeared after the tenth response in one component and after the fiftieth response in another. Pause duration was shortest with no time-out, longest with the time-out late in the ratio. In time-out components, response rate was low preceding the timeout, high immediately following it. The total time from rsponse initiation to subsequent reinforcement was highly predictive of pause duration. Immediate repetition of a component generally produces shorter pausing than when it was preceded by a different component.
\end{abstract}

A time-out (TO) is a signaled period of time associated with extinction (Ferster \& Skinner, 1957) during which reinforcement is unattainable (Leitenberg, 1965). It is generally signaled by a change in some exteroceptive stimulus within the operant chamber.

The present study involves the systematic introduction of TO during fixed-ratio (FR) performance. The FR, which provides reinforcement following a fixed number of responses, typically generates a period of pause followed by a rapid sequence of responses terminating with reinforcement. A major determinant of the pause duration is the length of the ratio itself. Some research (Neuringer \& Schneider, 1968) suggests that the time devoted to the sequence of responses to complete the ratio affects pause duration.

The intent of the present analysis is twofold. For one, we are concerned with pause duration as it is affected by the time involved in obtaining reinforcement. Our procedures included a multiple schedule of reinforcement in which an FR 60 was always employed. The three distinctively cued components included two with 10 -sec TOs regularly provided at different loci in the response sequence. Our second concern was an examination of the influence of the TO and its position, in the sequence of responses, on local variations in response rate.

\section{METHOD}

\section{Subjects}

Three male white Carneaux pigeons, between 2 and 3 years old, were maintained at $80 \%$ of their free-feeding weights. All

This research was supported in part by a Grant from The City University of New $\because$ ork Faculty Research Award Program. Reprints may be obtained from Donald E. Mintz, Department of Psychology, City College of New York, New York, New York 10031. birds were experimentally naive at the start of the experiment.

\section{Apparatus}

A standard Lehigh Valley Electronics two-key operant chamber for pigeons was used. Only the key to the left of the food hopper was operative for purposes of this study. Temporal data were recorded on Pressin printing counters, mounted in Scientific Prototype control housings and pulsed at $10 \mathrm{~Hz}$ by a Scientific Prototype reed clock. The test chamber was housed in a sound-attenuating enclosure, and white noise was provided for further masking. Closure of the response key required a force of approximately $.2 \mathrm{~N}$.

\section{Procedure}

The three pigeons were shaped to peck the left key of the operant chamber, transilluminated white. Subsequent training involved systematic increases in the responses required for reinforcement until responding on FR 60 in the presence of the white key stablized. The birds then received 2 days of training with key light either red, green or white, changing after each reinforcement.

During training on the multiple schedule, key color was programmed in a mixed sequence, each color being presented with approximately equal frequency and juxtaposition to the other colors. However, no more than two successive presentations of the same color were provided. In the three-ply multiple FR 60 schedule the components were: FR 60; FR 60 with a 10 -sec TO contingent upon response 10 (designated as early); FR 60 with a 10 -sec TO contingent upon response 50 (designated as late). Any response during the 10-sec TO recycled the timer so that a minimum of $10 \mathrm{sec}$ had to elapse with no responding. The TO was signaled by the unilluminated response key; the house light remained illuminated throughout the session. Key-color correlations with the schedules were counterbalanced across birds.

Reinforcement was $3.0-\mathrm{sec}$ access to grain. Initial training involved 60 reinforcements per session; 66 reinforcements were given during multiple-schedule training. The birds were exposed to the multiple schedule for 29 days. Days 20 and 29 served as criterion days, and the data reported is based on all performance during these 10 days. 
Table 1

Median Pause Duration (in Seconds) in FR 60 Related to the Schedule in Effect and the Immediately Preceding Schedule

\begin{tabular}{llccc}
\hline & & \multicolumn{3}{c}{ Present Schedule } \\
\cline { 3 - 5 } & Preceding & No & TO & TO \\
& Schedule & TO & (R10) & (R50) \\
\hline \multirow{4}{*}{ P 1 } & No TO & 4.8 & 12.3 & 28.3 \\
& TO (R10) & 5.8 & 6.4 & 28.1 \\
& TO (R50) & 6.8 & 11.8 & 8.4 \\
& Overall & 5.5 & 10.7 & 24.9 \\
P 2994 & No TO & 2.1 & 11.4 & 37.8 \\
& TO (R10) & 2.3 & 5.8 & 31.4 \\
& TO (R50) & 2.3 & 12.3 & 17.4 \\
& Overall & 2.1 & 10.5 & 26.9 \\
& No TO & 3.4 & 12.0 & 23.2 \\
& TO (R10) & 3.1 & 6.9 & 14.5 \\
& TO (R50) & 3.3 & 11.8 & 5.0 \\
& Overall & 3.3 & 10.2 & 14.9 \\
\hline
\end{tabular}

\section{RESULTS}

Table 1 shows the median pause generated by each component of the multiple schedule. These data also provide an analysis in terms of the component immediately preceding the one during which the pause was measured. Pausing was defined as the time from the termination of reinforcement until the first response of the FR cycle.

A clear hierarchy of pause duration is evident, the no-TO component generating brief pauses, the early-TO showing intermediate duration pauses, and the longest pauses occuring in late-TO schedule. In addition, an interaction between the present and preceding condition is suggested. Except for P 7871's no-TO data, the shortest pause within a condition is shown when that component schedule is preceded by itself. This suggested interaction has been repeatedly replicated by Barowsky (1973).

Response rate during the FR run was measured by recording the time to complete specified segments of the ratio. Figure 1 shows those data which characterize local rate variations related to the different schedules. The plotted points are means of 10 daily medians. In general, the least within-cycle variation is shown by the no-TO condition. All three birds show relatively large rate increases immediately following TO in the two schedules in which TOs were employed, P 1's early-TO data a minor exception. For this subject, the rate during the first 10 responses in the early-TO schedule was already the highest shown for the three schedules.

The general pattern suggests an initial rate acceleration for all conditions. This generally corresponded to the frequently observed stagger/start in which a few responses of long interresponse time (IRT) precede a shift to relatively short IRTs. Where TOs were employed, a relatively low rate persisted until the TO occurred and was followed by a high rate. The post-TO
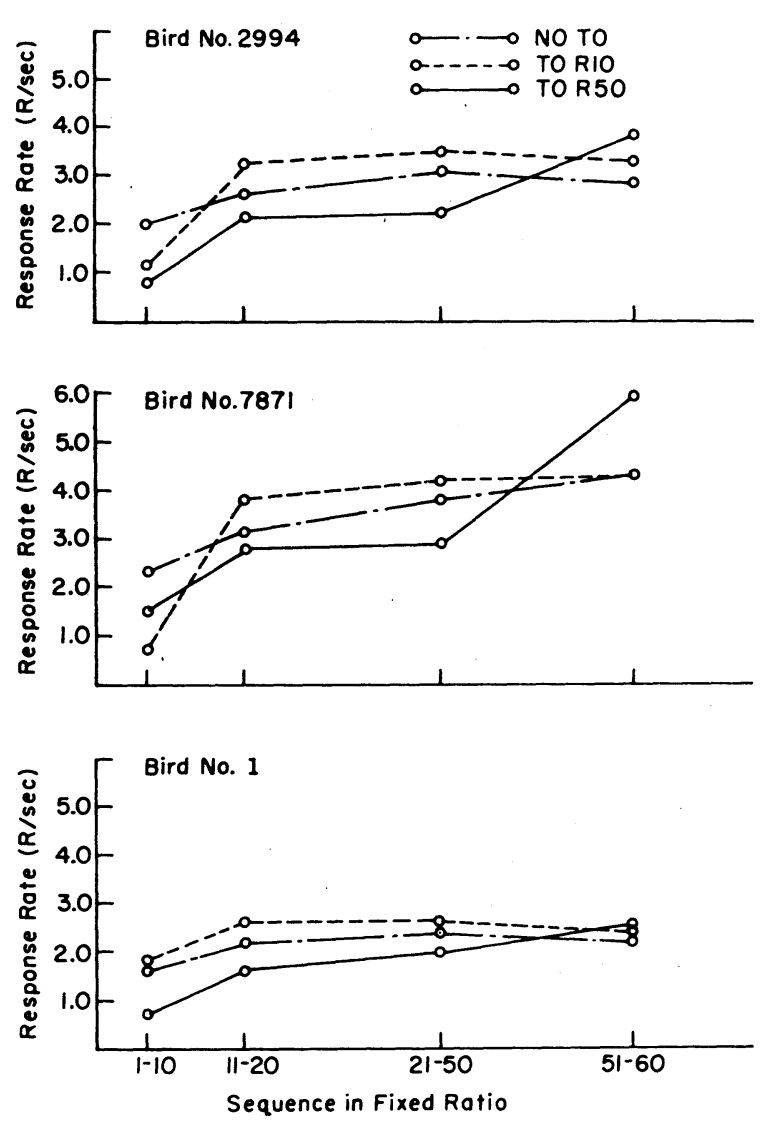

Figure 1. Median response rate during successive segments of the fixed-ratio cycle.

rate, in fact, was invariably higher than the corresponding rate in the no-TO component.

In Figure 2 a scattergram shows the relation between pause duration and time of involvement (TOI). TOI includes all time from initiation of the chain of behavior required for reinforcement until reinforcement is obtained. It subsumes both $\mathrm{TO}$ and response time. Both across and within birds a clear positive correlation is evident. A linear function is well approximated when pause duration is plotted logarithmically suggesting an exponential increase in pause duration with longer TOI. The regression equation for the combined data is log $\mathrm{Y}=.041 \mathrm{x}-.33$.

\section{DISCUSSION}

Pause duration preceding an FR run relates to the time from initiation of responding until the advent of reinforcement. Those conditions with longer TOIs generally produce longer pausing. In most FR procedures, the TOI is closely correlated with FR lengths and it is difficult to attribute pause duration to time, as opposed to response number. The present study, however, achieves well differentiated pausing in schedules with constant response requirements through some interesting consequences of an interposed TO. A similar effect has been reported by Neuringer and Schneider (1968) in a procedure aimed at keeping response number in FR constant, while manipulating the time to 


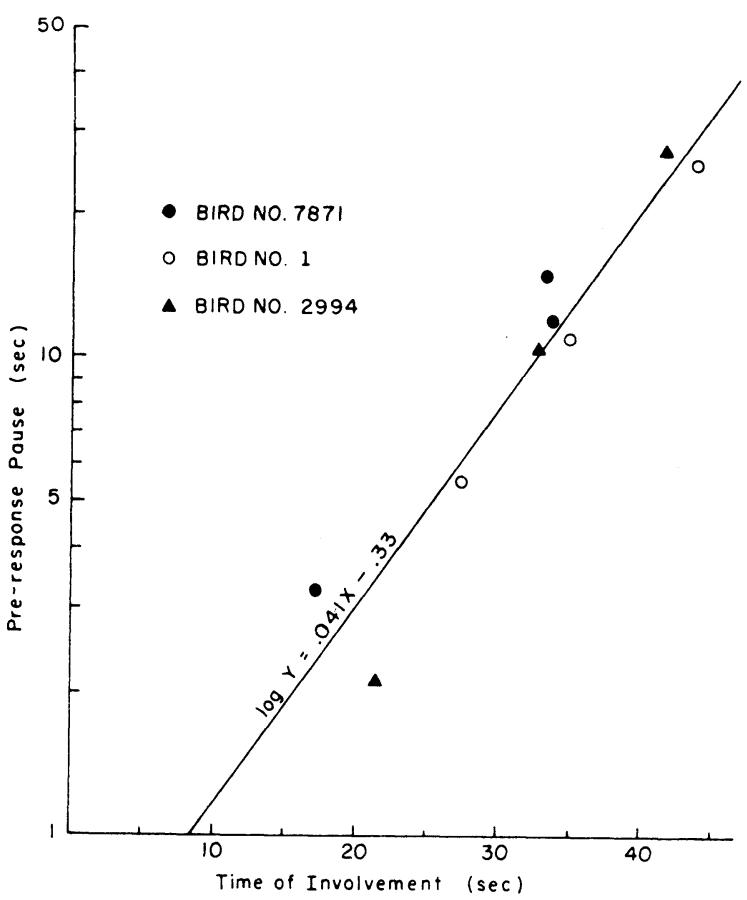

Figure 2. Scattergram of pause duration and time of involvement (TOI). TOI subsumes all time from the first response to subsequent reinforcement. The regression line is the least squares best fit for all plotted points.

complete the FR cycle. Time correlated determinants of pause duration are also suggested by Kileen (1969) and Zeiler (1970, 1972).

The major effect of the TO in the present study is to produce relatively low rates of response prior to the TO, and facilitated responding following it. The net consequence is a longer TOI the later the TO is introduced. Both the local rate variations and the duration of the TO itself affected TOI which in turn was highly predictive of pausing.

The present data also add more evidence that when FR length is reliably cued, the primary determinant of pause duration is the forthcoming ratio (Mintz, Mourer, \& Gofseyeff, 1967; Griffith \& Thompson, 1972). The interaction with the condition preceding the pause is less clear. The present, and subsequently replicated (Barowsky, 1973), evidence indicates that repetition of a condition leads to relatively shorter pausing. This facilitation might relate to the more recent (immediately preceding) reinforcement of responding in the presence of the particular stimulus cueing the repeated schedule. However, the validity of such a "recency" phenomenon requires demonstration in procedures more explictly aimed at its analysis.

\section{REFERENCES}

Barowsky, E. The effect of time-out locus and duration upon fixed-ratio responding in a multiple schedule. Unpublished doctoral dissertation, City University of New York, 1973.

Ferster, C. B., \& Skinner, B. F. Schedules of reinforcement. New York: Appleton-Century Crofts, 1957.

Griffiths, R. R., \& Thompson, T. Pausing in ratio schedules a function of upcoming ratio size. Proceedings of the 80th Annual Convention, American Psychological Association, $1972,7,753-754$.

Kileen, $P$. Reinforcement frequency and contingency as factors in fixed-ratio behavior. Journal of the Experimental Analysis of Behavior, 1969, 12, 391-395.

Leitenberg, $H$. Is time-out from positive reinforcement an aversive event? Psychological Bulletin, 1965, 65, 428-441.

Mintz, D. E., Mourer, D. J., \& Gofseyeff, M. Sequential effects in fixed-ratio postreinforcement pause duration. Psychonomic Science, 1967, 9, 387-388.

Neuringer, A. J., \& Schneider, B. A. Separating the effects of interreinforcement time and number of interreinforcement responses. Journal of the Experimental Analysis of Behavior, $1968,11,661-667$.

Zeiler, M. Time limits for completing fixed ratios. Journal of the Experimental Analysis of Behavior, 1970, 14, 275-286.

Leler, M. Time limits for completing fixed ratios. II stimulus specificity. Journal of the Experimental Analysis of Behavior, $1972,18,243-251$

(Received for publication October $3,1974$. 